\title{
Autonomic Agents for Survivable Security Systems
}

\author{
Roy Sterritt, Edward Hanna, and Patricia O'Hagan \\ ${ }^{1}$ University of Ulster, chool of Computing and Mathematics, \\ Jordanstown Campus, Northern Ireland \\ R.Sterritt@ulster.ac.uk \\ ${ }^{2}$ Core Systems, Belfast, Northern Ireland \\ Edward@coresystems.biz, Patricia@coresystems.biz
}

\begin{abstract}
Autonomic Systems are essentially about creating self-managing systems based on the biological metaphor of the non-conscious acting autonomic nervous system. The Autonomic initiative has been motivated by ever increasing complexity and total cost of ownership of today's system of systems. Autonomicity also offers inroads in terms of fault-tolerant computing and assisting in creating survivable systems. This paper examines the relevant technologies including Agents for engineering autonomicity and survivability in a secure location biometric system.
\end{abstract}

\section{Introduction}

The case has been well presented in the literature for the need to create self-managing systems due to the complexity problem, the total cost of ownership, or to provide the way forward to enable future pervasive and ubiquitous computation and communications [1][2][3][4]. Another aspect for self-management is to facilitate survivable systems. To enable self-management (autonomicity) a system requires many self properties (self-* or selfware), such as self-awareness. This paper first looks at autonomic management, it then describes a deployed critical security system that requires built in survivability and autonomicity. The paper then briefly examines related work, namely agents and clusters before going on to present an agent based approach to deal with providing self-management redundancy in the system and avoid the traditional updating issues with failover servers.

\section{Autonomic Management}

Computing systems are expected to be effective. This means that they serve a useful purpose when they are first introduced and continue to be useful as conditions change. Responses taken automatically by a system without real-time human intervention are autonomic responses [5]. The autonomic concept is inspired by the human body's autonomic nervous system. By analogy, humans have good mechanisms for adapting to changing environments and repairing minor physical damage. The autonomic nervous system monitors heartbeat, checks blood sugar levels and keeps the body temperature normal without any conscious effort from the human. This biological autonomicity is influencing a new paradigm for computing to create similar self- 
management within systems (Autonomic Computing, Autonomic Communications and Autonomic Systems). There is an important distinction between autonomic activity in the human body and autonomic responses in computer systems. Many of the decisions made by autonomic elements in the body are involuntary, whereas autonomic elements in computer systems make decisions based on tasks chosen to delegate to the technology [6].

Many branches of computer science research and development will contribute to progress in autonomic computing. In particular, it brings together work in software engineering and artificial intelligence. Research on dependable systems should be especially influential, as dependability covers many relevant system properties such as reliability, availability, safety, security, survivability and maintainability [7],[8].

In the late 1990s DARPA/ISO's Autonomic Information Assurance (AIA) programme studied defence mechanisms for information systems against malicious adversaries. The AIA programme resulted in two hypotheses; (1) fast responses are necessary to counter advance cyber-adversaries and (2) coordinated responses are more effective than local reactive responses [5]. These hypotheses may provide general guidance for creating autonomic survivable systems.

'Autonomic' became mainstream within Computing in 2001 when IBM launched their perspective on the state of information technology [1]. IBM defined four key self properties: self-configuring, self-healing, self-optimizing and self-protecting [6]. In the few years since, the self- $x$ list has grown as research expands, bringing about the general term selfware or self-*, yet these four initial self-managing properties along with the four enabling properties; self-aware (of internal capabilities and state of the managed component), self-situated (environment and context awareness), selfmonitor and self-adjust (through sensors, effectors and control loops), cover the general goal of self management [8].

The influence of the autonomic nervous system (ANS) may imply that the Autonomic Computing initiative is concerned only with low level self-managing capabilities such as reflex reactions. This fits with AIA perspective, other layered architectures such as; reaction-routine-reflection [9]; data-management/controlknowledge planes [10]; hardware-cyber-mission planes [5]; autonomicselfware-autonomous [11]. Yet within the autonomic research community the vision behind the initiative is an overarching goal of system-wide policy-based selfmanagement where a human manager will state a business-critical success factor and the ICT systems will take care of it, self-configuring and self-optimising to meet the policies, and self-protecting and self-healing to ensure the policies are maintained in light of changing circumstances. It may be reasoned that due to our ANS we are freed (non-conscious activity) from the low-level complexity of managing our bodies to perform high-level complex tasks. Similarly, for Computing to develop further and provide equivalent high-level system-wide tasks, necessitates a corresponding lowlevel 'non-conscious' architecture. As such, increasing this initiative will converge and cross-influence the fields of ubiquitous and pervasive computing.

\section{The Core Survivable Security System}

Highly secure locations, such as research labs and law enforcement correction centers, are seeking to utilize the latest technology in biometrics such as iris and finger print 
technologies to allow and restrict access and movement around their locations [12]. Architectural requirements for such systems dictate that the architecture should be secure, fault-tolerant, and non-exploitable [5]; that the system must meet the security policies of the organization and it must accommodate different security infrastructures; the system must remain robust and secure when faults occur, both random faults caused by the failure of system elements and to malicious faults caused by a deliberate attack on the system; that communication must be reliable so that defensive components remain fully functional even in the face of an attack on the infrastructure; and that it must not be possible for an attacker to exploit defensive components to effect an undesirable action, for example, an attacker or random fault must not be able to trigger a response that causes the system to unnecessarily deny legitimate users access and movement nor the opposite allow non-legitimate users access and movement around the location [5].

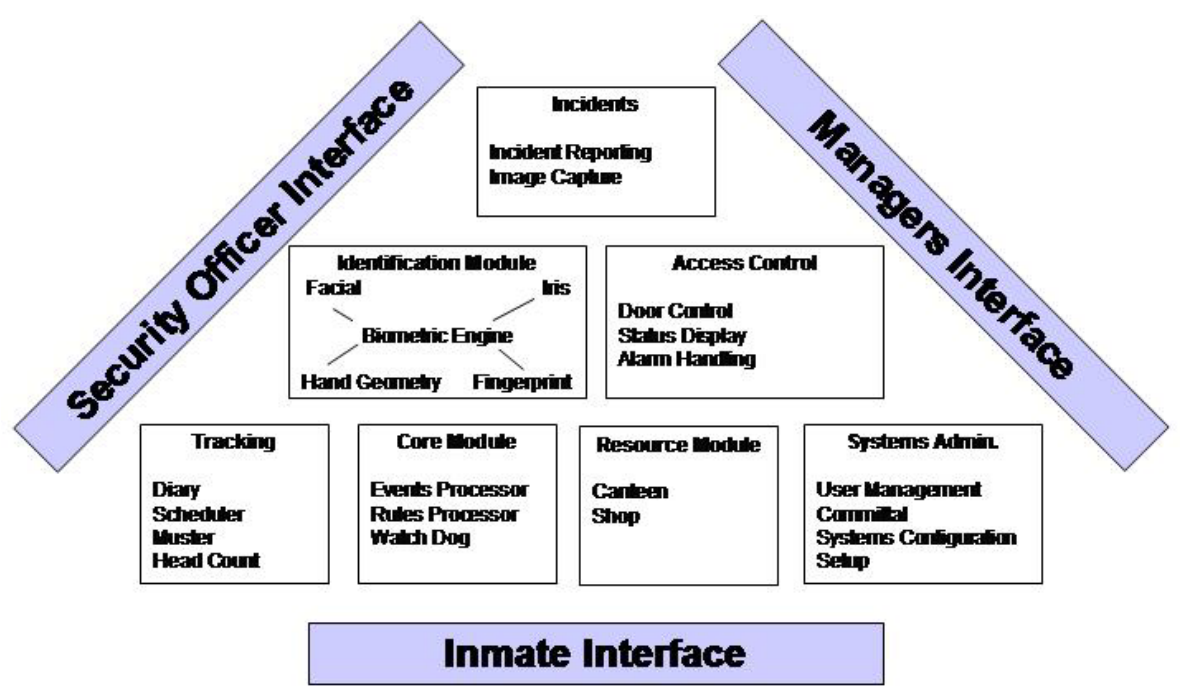

Fig. 1. Core Security System - High Level Architecture

Figure 1 depicts a high level architecture for access control to a correction center secure location incorporating such identification technologies as biometrics [12]. The implication of these multiple components is that the system becomes more complex, a theme the Autonomic initiatives aim to address. In this situation the self-healing and self-protections aspects are more critical to the system. The continuous monitoring of the system health through control loops matches the architecture needs. Research within the Autonomic field such as self-healing and micro rebooting can assist in creating a survivable environment [8]-[14].

Figure 2 illustrates the Survivable Secure System (SSS) architecture. It provides for an inmate tracking solution that delivers the information needed to manage the correction center. The system supports cell assignments and tracking of inmates' movements, various activities and events. The SSS is a modular system that will integrate with existing systems with the general concept being to monitor people 
within a defined space and manage their movements, access to goods, services and privileges. A predefined set of rules can be applied at any level to limit movement, interaction between individuals or access to resources.

A history will build up of activity for each individual defined to the system. Biometric technology is used to uniquely identify prisoners. This makes it possible to provide inmates with personal information and enable them to request services through the system.

\subsection{Survivability}

Survivability is achieved within the system through the use of Watchdog, heartbeats, micro-reboots, automated failover and a dual redundant ring architecture.

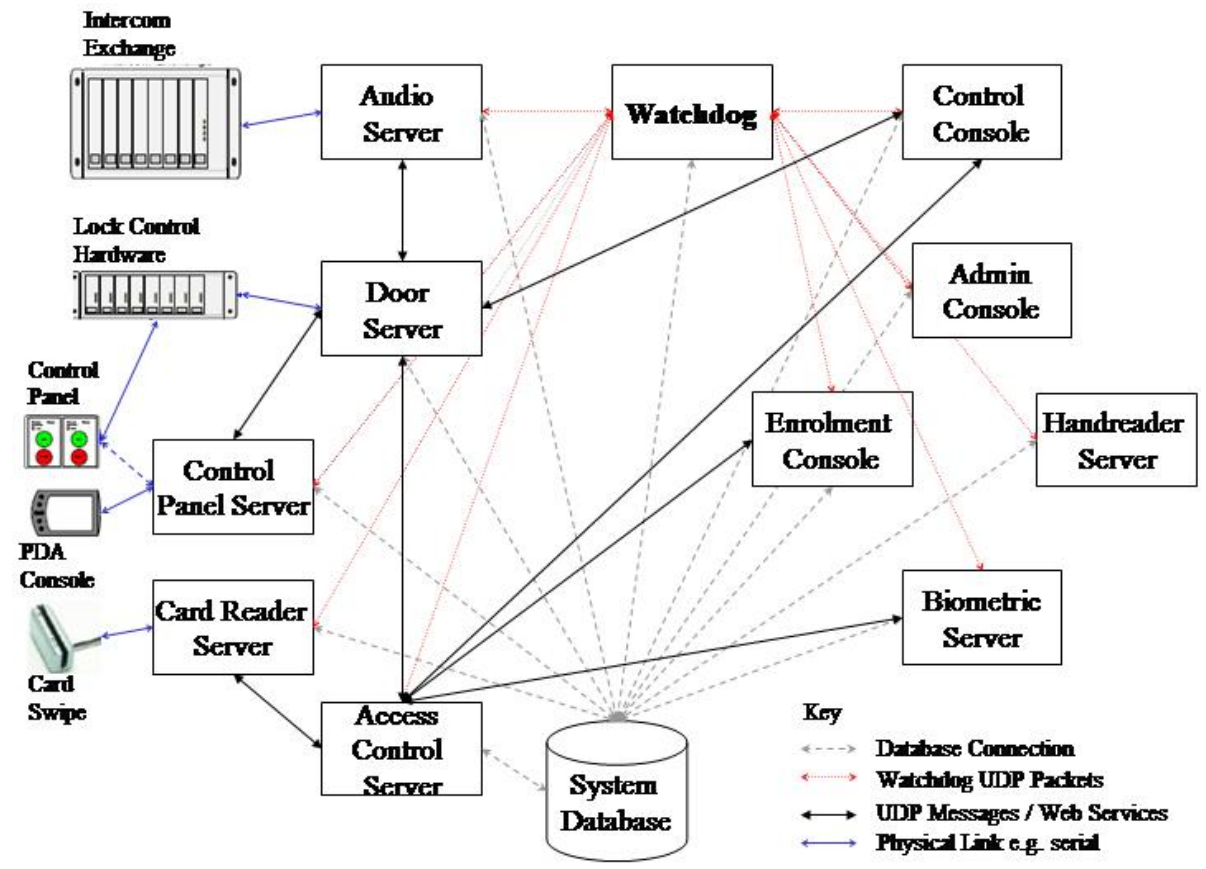

Fig. 2. Core Security System - Component Architecture

\subsubsection{Watchdog}

Watchdog is essentially status monitoring software, providing a service that monitors the status of a specific list of devices/programs across the network. This status information is sent to registered consoles where it is displayed in a graphical format representing the system. From the graphic layout users are shown the current system status and alerted to any problems. This enables the user to initiate pre-programmed remedial action. It is also possible to have the Watchdog perform automatic responses to specific problems. 
The Watchdog communicates with hardware devices either using standard pings or by using SNMP (Simple Network Management Protocol) messages. By using a standard ping the Watchdog can only ascertain if the device is replying or not. By using SNMP the Watchdog can collect more detailed information about the device, in the case of SNMP enabled switches (e.g. Netgear) individual links can be monitored. For software applications, the Watchdog sends heartbeat (Are you alive?) messages to which the application replies giving its status. The heartbeat messages also contain the status of other applications which have a functional relation to the application being polled. This additional information can be used by the application to make decisions on how to operate if a required function of the system is not working correctly.

If a problem is detected in an application the Watchdog can kill the faulty application and restart it. If a problem is detected in hardware the Watchdog can restart the device via software controlled power management.

\subsubsection{Heart-Beats}

Essentially, one view of the goal of autonomic computing is to create robust dependable self-managing systems [8]. To facilitate this aim, fault-tolerant mechanisms such as a heart-beat monitor ('I am alive' signals) and pulse monitor (urgency/reflex signals) may be included within the autonomic element [9][2]. The notion behind the pulse monitor (PBM) is to provide an early warning of a condition so that preparations can be made to handle the processing load of diagnosis and planning a response, including diversion of load. Together with other forms of communications it creates dynamics of autonomic responses [15] - the introduction of multiple loops of control, some slow and precise, others fast and possibly imprecise, fitting with the biological metaphor of reflex and healing [9].

\subsubsection{Micro-rebooting}

One promising avenue of research for self-healing (in particular in software) is microrebooting [13]. In this approach components at varying granularity levels are designed to be crash only, that is if they are not functioning correctly, self-healing is simply a reboot.

Since rebooting can be expensive causing non trivial service disruption or downtime (even when failover and clusters are employed) the key is to separate process recovery from data recovery and to achieve fine grained rebooting; i.e. components as opposed to applications or even systems [13].

\subsubsection{Redundant Ring Architecture}

Survivability is achieved within the network communications through a dual redundant fibre ring architecture. This is achieved by using Netgear layer 2 managed switches. For an Ethernet network to function properly, only one active path can exist between two stations. Multiple active paths between stations cause loops in the network. If a loop exists in the network topology, the potential exists for duplication of messages. To provide path redundancy on Ethernet networks the Spanning-Tree Protocol can be used. Spanning-Tree Protocol forces certain redundant data paths into a standby (blocked) state. If one network segment in the Spanning-Tree Protocol becomes unreachable, the spanning-tree algorithm reconfigures the spanning-tree topology and re-establishes the link by activating the standby path. This reconfiguration mechanism is transparent to the machines on the network. The blocked standby paths are illustrated as lighter grey connection in Figure 3. 


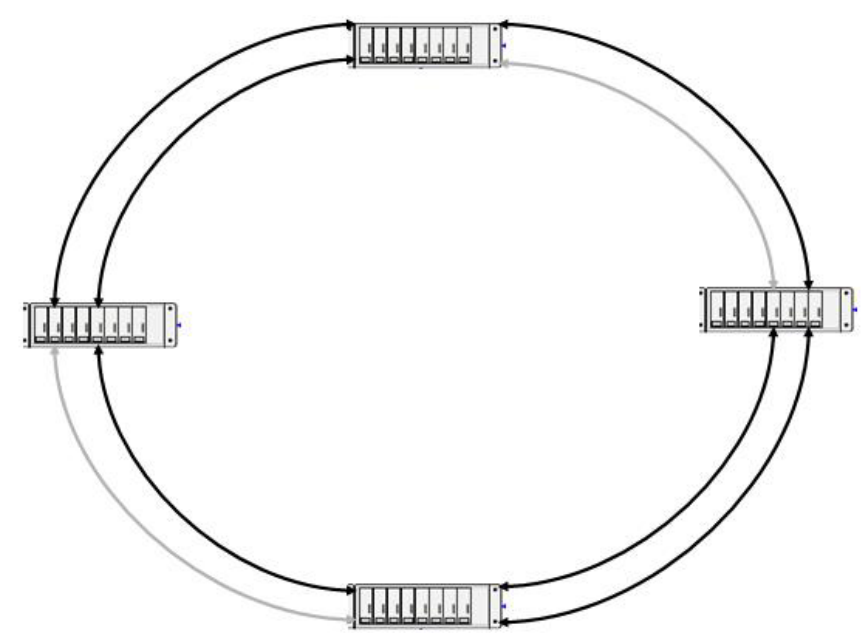

Fig. 3. Dual Redundant Ring Architecture

\subsubsection{Failover/Load Balancing}

The major server applications, illustrated in Figure 2 exist as multiple instances running on different machines. The load is balanced between these instances so that in the event of a machine failure there is minimal impact on the system. Local fail safe has been built into the access control system so that under extreme fault conditions the access control system will still function in a limited capacity.

\section{Related Work}

Autonomic Computing is dependent on many disciplines for its success; not least of these is research in agent technologies. At this stage, there are no assumptions that agents have to be used in an autonomic architecture, yet the desired self-* properties can benefit from the nature of agents, namely; autonomy, reactivity, sociality and proactivity. Also, as in complex systems there are arguments for designing the system with agents [16], as well as providing inbuilt redundancy and greater robustness [17], through to retrofitting legacy systems with autonomic capabilities that may benefit from an agent approach [18].

Emerging research ranges from autonomic architecture containing autonomic managers as agents themselves, a self-managing cell [19], containing functionality for measurement and event correlation and support for policy-based control, to alternative rational models, such as state-feeling-action [20], that may better suit autonomic systems than traditional DBI models. Autonomics is also finding its way into agent research, for instance semantic web agents [21].

Cluster computing, whereby a large number of simple processors or nodes are combined together to apparently function as a single powerful computer, has emerged as a research area in its own right. Traditionally, Massively Parallel Processing (MPP) computer systems have been used to meet high performance computing requirements. MPP computers may contain hundreds or thousands of processors within a single computer system. Typically, upgrading such systems requires a 
complete rebuild of the system. They are, however, relatively simple to manage, and they certainly perform very well. A recent trend in high performance computing research has been to find new approaches to overcome the cost and scalability issues associated with MPP systems, such as clusters and grids.

The Cluster approach offers a relatively inexpensive means of providing a faulttolerant environment and achieving significant computational capabilities for highperformance computing applications. However, the task of manually managing and configuring a cluster quickly becomes daunting as the cluster grows in size. Autonomic computing is also being researched to provide self-managing cluster systems, for instance the Autonomic Cluster Management System (ACMS) [22] that exploits autonomic properties and agents in automating cluster management.

\section{Autonomic Management with Agents}

A major issue with providing redundancy and automated failover is the expense in keeping the back-up servers up-to-date. When you also consider the SSS environment where there are many types of servers this becomes particularly critical. If one utilizes autonomic agents to design the self-management system, then such properties as agent cloning may be utilized to provide up-to-date back-up redundant servers.

This section considers the autonomic self-managing survivable system as a mobile agent system, much the same as the ACMS [22]. The system is composed of a number of agent processes, representing both the required servers (control panel server, door server, audio server and so on) and the necessary autonomic management agents, communicating across a network of nodes. The system consists of four types of agents, each with functionality implementing autonomic system properties, namely the General Server Agents (GA), Health Agents (HA), Optimization Agents (OA), and Configuration Agents (CA).

One configuration to provide the survivable autonomicity is that the system is comprised of two Configuration Agents, two Health Agents, and one Optimization Agent per implementation, as well as two General Agents per node (primary and redundant secondary). Each general agent is designed to be specific-purpose, and to perform a particular task (the servers in the SSS). The community of agents collaborates to achieve a common goal, specifically providing autonomic management of the system, while simultaneously maximizing performance by implementing load-balancing techniques on the system. Figure 4 illustrates the architecture of the proposed system.

The agents function includes a heartbeat (sending of a periodic 'I am alive' signal) [9], to facilitate fault tolerance and the provision of localized fail-over on the node; i.e., instead of the traditional approach of the CA noticing an agent has failed (through polling) on a remote host and instructing the switch over to the secondary agent on that remote host, this can occur locally via the secondary agent monitoring the heartbeat from the primary agent and thus providing a tighter and situated reflex reaction upon failure.

The primary and secondary CAs will also utilize the same mechanism with heartbeats between them (typically on separate hosts to increase fault tolerance). Selfhealing is provided for explicitly through a health agent. Its function, in collaboration with the CAs and OAs, will be to monitor vital signs on the hosts in an attempt to predict if a host is having difficulties and a failure is imminent. 

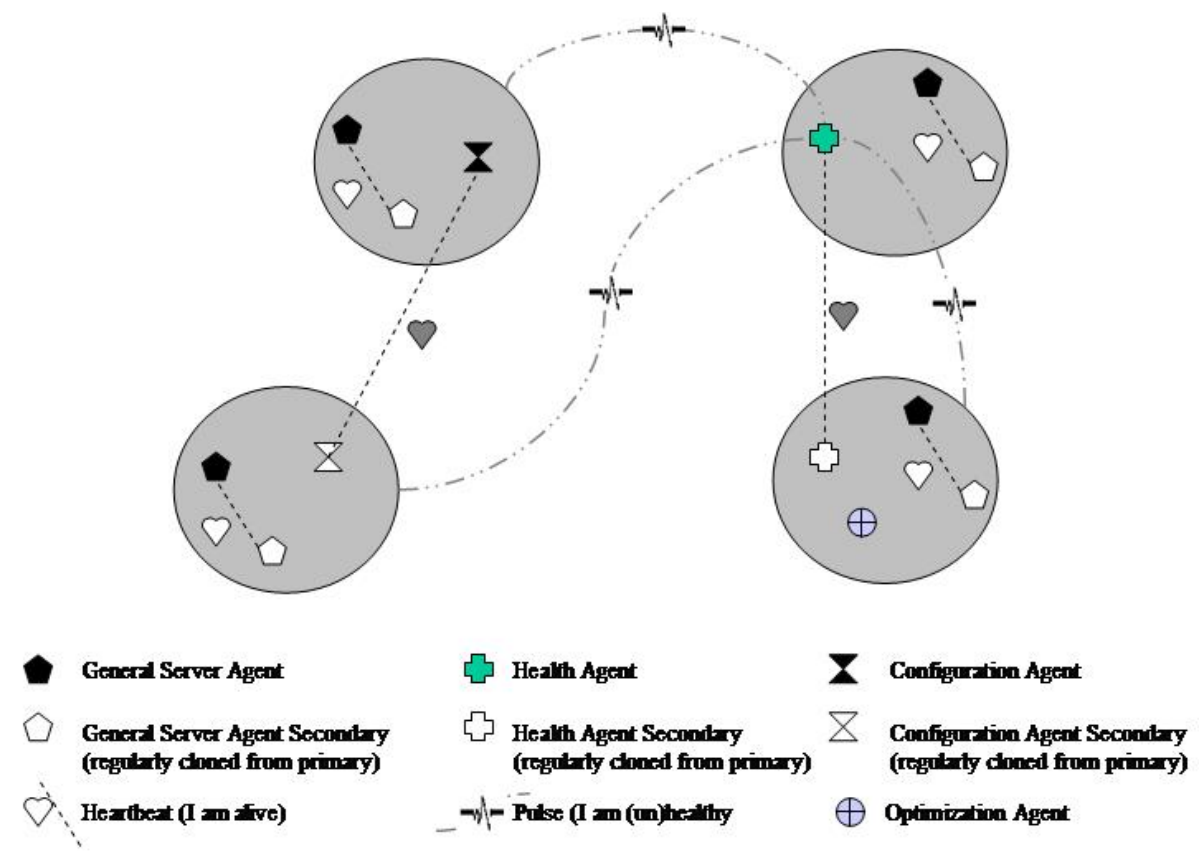

Fig. 4. Autonomic Agents Survivable Self-Management System

The health agent will be facilitated by pulse monitoring - the extension of heartbeat monitoring from 'I am alive' signals to include health information within the signal, akin to measuring the pulse [9]. In this scenario the local agents may failover with a new secondary agent being created, yet if this starts to occur frequently this may indicate the host itself is unstable. The health agent can monitor developing scenarios and work with the configuration agent to avoid allocating work to unstable hosts [22].

\section{Conclusion}

This paper has highlighted that Autonomic Systems are not just motivated by concerns over complexity and total cost of ownership, but also extending faulttolerance to create survivable systems by utilizing self-* properties.

We have described a deployed Biometric Identification and Tracking System incorporating survivability and autonomicity, used in law enforcement and correction centers with the aim to monitor people within the establishment in terms of movements, interactions and access to resources.

In these environments it is simply not acceptable for failure at the system level and as such the architecture must have multiple mechanisms to ensure survivability. In the development of the architecture it has been endeavored to incorporate into its design the AIA hypotheses of fast and coordinated responses. The paper also considered a proposed mobile agent solution to increase the flexibility and survivability within the system. 
Future extensions of this work will include adding layers above the survivable autonomic system such as Knowledge-based alerting to incidents occurring with the correction center.

\section{Acknowledgements}

The development of this paper was supported by a Knowledge Transfer Partnership project funded by the UK Government's Department of Trade and Industry (DTI). Core Systems' development project is partly supported by InvestNI. The wider context of the Autonomic Systems research is supported at University of Ulster by the Computer Science Research Institute (CSRI) and the Centre for Software Process Technologies (CSPT), funded by Invest NI through the Centres of Excellence Programme, under the EU Peace II initiative.

\section{References}

1. P. Horn, "Autonomic computing: IBM perspective on the state of information technology," IBM T.J. Watson Labs, NY, 15th October 2001.

2. R. Sterritt, "Towards Autonomic Computing: Effective Event Management", Proc. IEEE/NASA SEW, Greenbelt, MD, Dec. 2002.

3. JO Kephart, DM Chess. "The Vision of Autonomic Computing", Computer, 36(1):41-52, 2003.

4. R. Sterritt, "Autonomic Computing", Innovations in Systems and Software Engineering, Vol. 1, No. 1, Springer, pp 79-88, 2005

5. SM Lewandowski, DJ Van Hook, GC O'Leary, JW Haines, LM Rossey, "SARA: Survivable Autonomic Response Architecture", DARPA Information Survivability Conference and Exposition II Proceedings, Vol. 1, pp. 77-88, June 2001.

6. IBM, “An architectural blueprint for autonomic computing", 2003.

7. B. Randell, "Turing Memorial Lecture - Facing Up to Faults", Comp. J. 43(2), pp 95-106, 2000.

8. R Sterritt, DW Bustard, "Autonomic Computing: a Means of Achieving Dependability?", Proc IEEE Int. Conf. on the Engineering of Computer Based Systems (ECBS'03), Huntsville, Alabama, USA, April 7-11 2003, pp 247-251.

9. R. Sterritt, "Pulse Monitoring: Extending the Health-check for the Autonomic GRID", Proceedings of IEEE Workshop on Autonomic Computing Principles and Architectures (AUCOPA 2003) at INDIN 2003, Banff, Alberta, Canada, 22-23 August 2003, pp 433-440.

10. D Clark, C Partridge, JC Ramming, JT Wroclawski, "A Knowledge Plane for the Internet", Proc. Applications, technologies, architectures, and protocols for computer communication, Karlsruhe, ACM SIGCOMM 2003

11. R. Sterritt, MG Hinchey, (Jun 2005) "SPAACE :: Self- Properties for an Autonomous \& Autonomic Computing Environment", Proceedings of Autonomic \& Autonomous Space Exploration Systems (A\&A-SES-1) at 2005 International Conference on Software Engineering Research and Practice (SERP'05), Las Vegas, June 27-30, CSREA Press, Pages 3-8

12. R. Sterritt, G. Garrity, E. Hanna, P. O'Hagan, "Survivable Security Systems through Autonomicity", 2nd NASA GSFC/IEEE Workshop on Radical Agent Concepts (WRAC II), 2005 
13. G. Candea, S. Kawamoto, Y. Fujiki, G. Friedman, A. Fox, Microreboot - A Technique for Cheap Recovery. 6th Symp Operating Systems Design and Implementation (OSDI), San Francisco, CA, December 2004

14. R. Sterritt, "Autonomic Networks: Engineering the Self-Healing Property", Engineering Applications of Artificial Intelligence, Vol. 17, No. 7, Elsevier, ISSN 0952-1976, Pages 727-739, Oct 2004

15. R. Sterritt, D.F. Bantz, "PAC-MEN: Personal Autonomic Computing Monitoring Environments," Proc IEEE DEXA 2004 Workshops - 2nd Int. Workshop on Self-Adaptive and Autonomic Computing Systems (SAACS 04), Zaragoza, Spain, Aug. 30 - 3 Sept., 2003.

16. N.R. Jennings, M. Wooldridge, "Agent-oriented Software Engineering," in J. Bradshaw (ed.), Handbook of Agent Technology, AAAI/MIT Press, 2000.

17. M.N. Huhns, V.T. Holderfield, R.L.Z. Gutierrez, "Robust software via agent-based redundancy," Second International Joint Conference on Autonomous Agents \& Multiagent Systems, AAMAS 2003, July 14-18, 2003, Melbourne, Victoria, Australia, pp 1018-1019.

18. G. Kaiser, J. Parekh, P. Gross, G. Valetto, "Kinesthetics eXtreme: An External Infrastructure for Monitoring Distributed Legacy Systems," Autonomic Computing Workshop - IEEE 5th Int. Active Middleware Workshop, Seattle, USA, June 2003.

19. E. Lupu, et al., EPSRC AMUSE: Autonomic Management of Ubiquitous Systems for eHealth, 2003.

20. Hu Jun, Gao Ji, Huang Zhongchao, Liao Beishui, Li Changyun, Chen Jiujun, A New Rational Model for Agent for Autonomic Computing, 2004 IEEE International Conference on Systems, Man and Cybernetics, pp 5531-5536, 2004.

21. V. Tamma, I. Blacoe, B. Lithgow Smith, M. Wooldridge "Introducing autonomic behaviour in semantic web agents", Proceedings of the Fourth International Semantic Web Conference (ISWC), Galway, Ireland, November 2005.

22. WF Truszkowski, MG Hinchey, R Sterritt, "Towards an Autonomic Cluster Management System (ACMS) with Reflex Autonomicity", Proc Workshop Reliability and Autonomic Management in Parallel and Distributed Systems (RAMPDS-05) at ICPADS-2005, Fukuoka, Japan, July 20-22, pp 478-482, July 2005 